\title{
Eye Controlled Mobile Robot with Shared Control for Physically Impaired People
}

\author{
Muhammad Wasim \\ Al-khawarizmi Institute of Computer Science, \\ UET Lahore, Pakistan \\ Javeria Khan \\ Al-khawarizmi Institute of Computer Science, \\ UET Lahore, Pakistan
}

\author{
Dawer Saeed \\ Al-khawarizmi Institute of Computer Science, \\ UET Lahore, Pakistan \\ Dr. Usman Ghani Khan \\ Al-khawarizmi Institute of Computer Science, \\ UET Lahore, Pakistan
}

\begin{abstract}
Physically impaired and disabled people are an integral part of human society. Devices providing assistance to such individuals can help them contribute to the society in a more productive way. The situation is even worse for patients with locked-in syndrome who cannot move their body at all. These problems were the motivation to develop an eye controlled robot to facilitate such patients. Readily available commercial headset is used to record electroencephalogram (EEG) signals for classification and processing. Classification based control signals were then transmitted to robot for navigation. The robot mimics a brain controlled wheelchair with eye movements. The robot is based on shared control which is safe and robust. The analysis of robot navigation for patients showed promising results.
\end{abstract}

Keywords-Locked-in syndrome; EEG; shared contol; eye controlled robot

\section{INTRODUCTION}

Brain Computer Interface (BCI) provides direct communication between computer and human brain by detecting electromagnetic brain signals and converting them into appropriate command signals[1]. The recorded brain activity can be used for assistive devices, gaming, and robotics. Signal recorded by brain is categorized as invasive or noninvasive. In Invasive BCIs electrical signals are recorded directly on or inside the cortex which require surgery to implant these electrodes. Different methods are used for recording these signals. Electrocorticogram $(\mathrm{ECoG})$ signals are recorded which involves electrical signals for its subsequent analysis[2]. Another method uses an array of multiunit electrode placed in the cortex to record the natural activity for small population of neurons[3]. Both methods have greater signal-to-noise ratio, but require very less training of the user and are suitable for restoring the damaged neurons of the patients[4]. In non-invasive BCIs electrical activity of brain is recorded by placing the electrodes on the scalp so it does not require surgery[5]. Non-invasive BCIs use various types of signals form brain as inputs, such as electroencephalograms (EEG), blood-oxygen-level-dependent (BOLD), deoxyhemoglobin concentrations, and magnetoencephalograms (MEG) which detects weak magnetic fields due to the current flow in the cortex[6]. EEG signals are most popular among all of them due to their low cost and convenient to use[7].

Recently robots are not only used in industry but also in daily life activities to provide assistance especially for the people suffering from disabilities like locked-in syndrome which is a neurodegenerative disorder [8]. In this syndrome person is fully aware of the environment but is not able to convey his/her commands so brain computer interface helps in providing a promising means to regain their mobility. Lockedin syndrome which leads to amyotrophic lateral sclerosis (ALS)[9] effects the motor neurons of the patients so the conventional EEG signal from motor imagery is not enough to control the robot which requires a lot of training and can be a tiring and stressful process. EEG signals from eye movements can provide aid for people suffering from such disorders. In previous work, electrooculogram (EOG) was used to detect eye movements which captures the corneal-retinal potential (CRP)[10]. The EOG varies from 0.05 to $3.5 \mathrm{mV}$ and is proportional to eye movements. Electrodes are placed near the frontal part of brain near the eyes to measure electrooculographic potential (EOG), which is a function of eye position with respect to the head[11]. The strength of signal captured by this method is good but it causes discomfort for the user.

BCI robots based on EEG signals are most widely used in robotics and in assistive devices. The objective of BCI-robots is to convert the human intentions into appropriate commands so robots can perform various complex tasks which allows user to control robots naturally without any external signal. BCI robots are categorized into two main classes as mobile robots and brain-controlled manipulators[12]. Recently due to the development in brain computer interface mobile robots have gain the attention of most researchers due to their ability to transport disabled people [13] [14] but at the same time these mobile robots require higher safety as they are designed to provide transportation for disabled people. To implement these robots in real life much higher accuracy is required.

In this proposed method EEG based non-invasive BCI is used to detect the eye movement. Instead of using conventional high cost multiple channel EEG systems, low cost non-invasive EEG system is presented. On the basis of 
the signals received by the physical movements of eye, the decisions are taken and hence the robot navigates accordingly.

The paper is organized in a way that related work discusses the previous methodologies used for brain signal extraction and shared control implementation. System model explains the overall proposed system and robot setup. Methodology section discusses the signal acquisition and its processing for the detection of eye movements and also the shared control implementation.

Robot movement under different scenarios explains the robot navigation under various situations. Experiments and results section describes the experiments designed to evaluate its performance and results are also shown. The conclusion section summarizes the results and discussions made in the paper. Finally the future works explains the future aspects of this work.

\section{RELATED WORK}

BCI is most widely used in real time applications like gaming [15], [16], virtual reality [17] and in robotics [18], [19], [20]. Apart from these applications BCI can provide assistance for the individuals suffering from severe mobility disorders such as brain controlled wheelchairs for people suffering from disabilities [21], [22], telepresence robots to provide aid for disabled persons[23], and exoskeletons [24],[25].

In the past image processing techniques were used to detect the eye movements [26]. Eye gaze tracking technique which consist of camera and computer, by computing the distance traveled by pupil, the movement of eye is tracked[27] videooculography systems (VOG)[28] or infrared oculography (IROG) [29] based on position of eye were also used. These previous techniques increase the complexity of the system but with the recent advancement in the brain computer interface researchers are now working on brain signals to capture the desire brain signal for BCI applications[30].

Apart from the signal extraction shared control anatomy is very important for all the assistive devices. It is very essential to develop such a design in which system is aware of the environment and capable of deciding that when to give control to human, machine or both[7]. Research work is being done to establish a shared control that can be implemented in real time environment. A key feature in these shared control design is the use of several assistance modes[23]. Most researchers [31], [32], [33] used autonomous approach in which the user only specify the destination and the navigation system plans a short and safe path.

Zhang[31] presented autonomous navigation in which user selects the destination by using P300 based BCI. Obstacle map is constructed in $2 \mathrm{D}$ with the help of webcam and laser range finder sensor and the way-points are generated using generalized Voronoi diagram. The research work [32] presented the autonomous approach in which user selects the destination point from the screen which displays real time scenarios constructed by a laser scanner which is transmitted to autonomous navigation system. It helps user to navigate in an unknown environment with the help of sensors. Once the destination is selected, user can relax which reduce the mental workload. Rebsamen and Cuntai Guan [33] also presented autonomous approach where paths are already defined and user can select only from these paths. This method require continuous update in the predefined paths in case of any change in environment. It is only suitable for a room equipped with webcams and not suitable for real time environment. Autonomous approach has a serious drawback that the user has no control on the robot and it does not work well in the situation where the environment stochastic so under these circumstances it is better to control the robot by the user and use semi-autonomous approach instead of autonomous approach.

Leeb and Robert [23] used semi-autonomous approach in which user control high level commands (turn left, right) while low level commands (avoiding obstacle) are implemented by the robot. Only two classes are used and the default behavior of the robot is to move forward with a constant speed. In case of repellers or attractors (representing obstacle and target) the motion of device changes in order to avoid them. In this proposed shared control semi-autonomous approach is implemented. Instead of using two classes, four classes (forward, backward, left, right) are used to give maximum control to the user. The default behavior of the robot is to stop in order to avoid any dangerous situations.

In this paper EEG is used to capture brain signal corresponding to the eye movements. Four classes are used for the movement of robot. Forward command is executed when the user lift his eyebrows. Left and right movement is implemented by the pupils movements to the left and right receptively. Eye blink is used to stop the robot. In order to differentiate from normal blinking of eye user have to blink three times. In this proposed shared control method semiautonomous approach is implemented to give the maximum control of robot to the user.

\section{SYSTEM MODEL}

The proposed BCI system consists of three main components: Signal acquisition unit which records electrical signals corresponding to the eye movements; the control unit which decides the desired motion commands on the basis of EEG signal received from EPOC headset; and the shared control unit ensuring the safety of user by avoiding obstacles. The block diagram of the system is shown in the figure 1 .

\section{A. Robot Setup}

The test robot is made from readily available and open source equipment to demonstrate the proof of concept. Short range ultrasonic sensors are used to detect the obstacles in front of robot. Each sensor covers a $15^{\circ}$ of range in front of it so to cover more range in front of the robot, three sensors have been used. While only one sensor has been used on rest of the three sides. To control the motor an H-Bridge is implemented in between the motors and microcontroller. The communication of the robot and control unit is done using Bluetooth. 


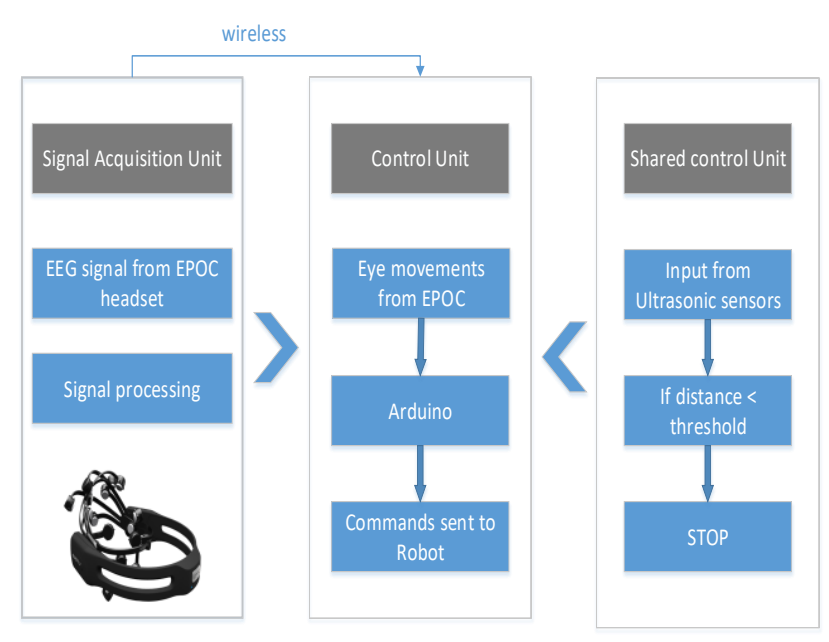

Fig. 1. Proposed Shared Control System Model

All the above mentioned setup is implemented using cheap and readily available components. An image of the developed robot is shown in figure 2 . The robot has custom made wheels which are developed in order to make it very stable and aligned to move in a straight path. The wheels are also light weight and already have an $\mathrm{O}$ ring which has reduced its surface friction.

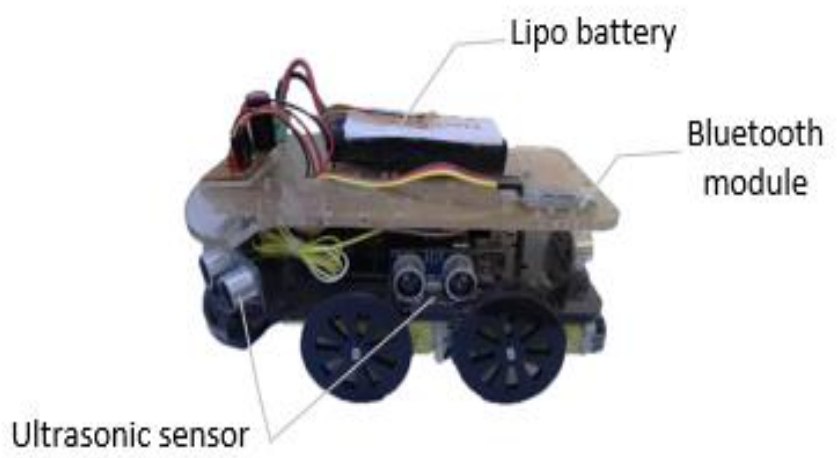

Fig. 2. Eye Controlled Mobile Robot

\section{METHODOLOGY}

The proposed system provides a method to detect the muscle activity of eyes through EEG signals which filtered the acquired signal for identifying eye movements. Emotiv API processes the acquired signals and performs classification. Once classified the control signals are transmitted to the robot. A high level system perspective is shown in the figure 3 .

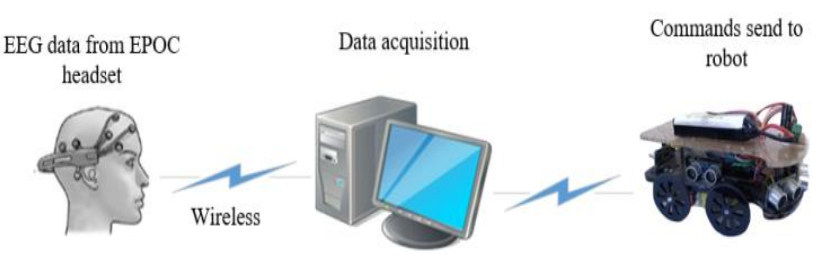

Fig. 3. System overview

\section{A. Signal Acquisition and Processing}

A widely used and commercially available wireless EEG headset Emotiv EPOC ${ }^{1}$ with 14 channels is used for BCI application. Software Development Kit (SDK) from Emotiv is also used for signal acquisition and classification. The application consists of three emotiv suites which are named as affectiv, cognitiv, and expressiv suite. These suites are developed to recognize the user's emotional state, thoughts and facial expressions respectively.

This experiment focuses on expressive suit which filters out the facial expression and hence eye blinks. The specific signals related to eye movement are classified from a wide range of facial expressions. This classification mainly uses the electroencephalogram (EEG) for eye blink and for the detection of muscle movements for looking right and left. Since all the users have same facial expressions so it does not need training. Sensitivity of the signal can be changed through the classifier. Certain facial expressions for some users are weak to detect so the sensitivity was tuned accordingly. Since every expression is a different expression so the sensitivity of each and every action was also fine-tuned accordingly.

\section{B. Shared Control and Robot Movement}

One of the most important and tedious task of controlling through $\mathrm{BCI}$ is its accuracy and high latency in processing the signals which can be tedious for the user some times. Also user's safety is very important. To ensure the user safety and to reduce the mental work load from user shared control is implemented. Shared control takes over the user in case of any obstacle is critically close. To implement the shared control, signal received from brain is classified into 4 classes (forward, left, right, and stop). The shared control implements the lower level control like obstacle avoiding, critical distance handling and collision prevention while the user controls the high level commands. The default behavior of the robot is to stop. To move the robot from its position either the obstacle has to be moving and the robot will counter for it accordingly or the robot must receive a signal from brain. As the robot receives signal, it keeps on moving in the respective direction until the user sends a signal again or an obstacle is detected. The flow chart of the algorithm implemented on the robot is also shown in the figure 4 .

\footnotetext{
${ }^{1}$ https://www.emotiv.com/epoc/.
} 


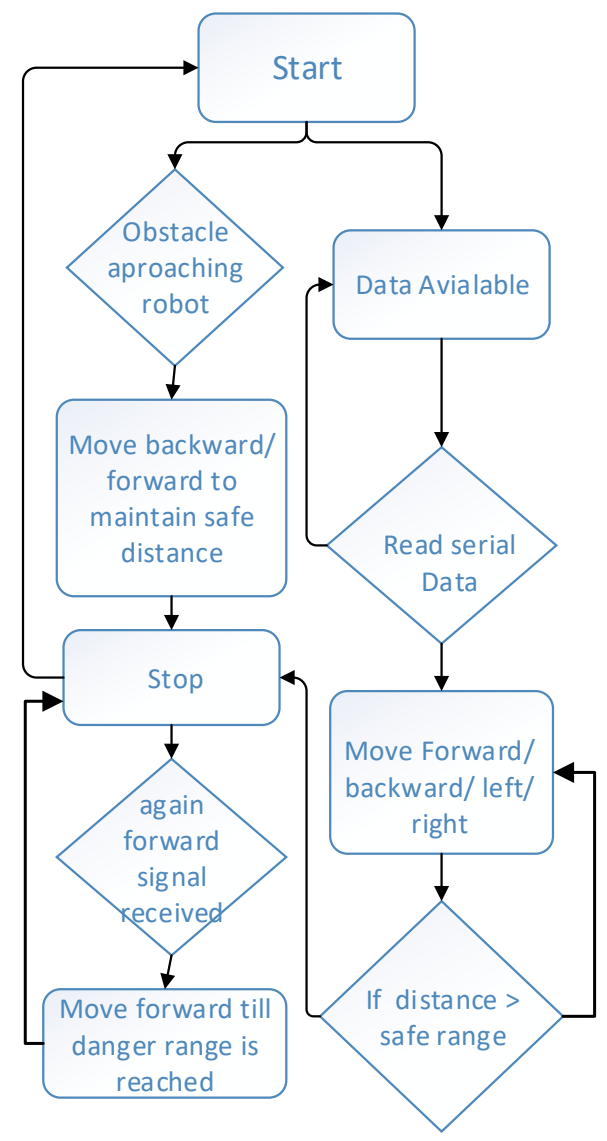

Fig. 4. Flow chart of the shard control algorithm.

\section{ROBOT MOVEMENT UNDER DIFFERENT SCENARIOS}

The shared control along with safety and other very promising features also comes with difficult control and working situations under different circumstances. The basic obstacle avoidance of the robot is shown is shown in figure 5 .

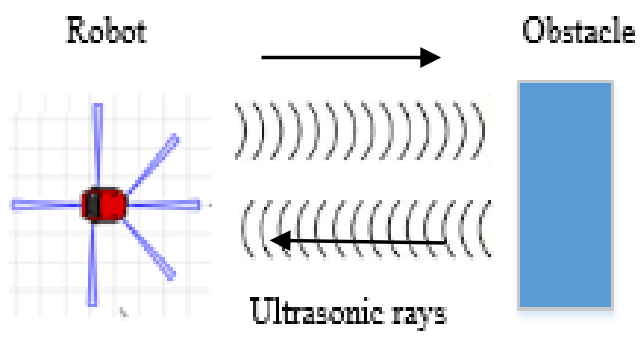

Fig. 5. Obstacle detection through ultrasonic sensor

The robot in presented work also behaves on different scenarios which are explained as follows:

\section{A. External Signal}

The robot moves forward as soon as the forward command is received from BCI. Whenever an object comes within the safe range the robot stops so that the safety of the user is maintained.

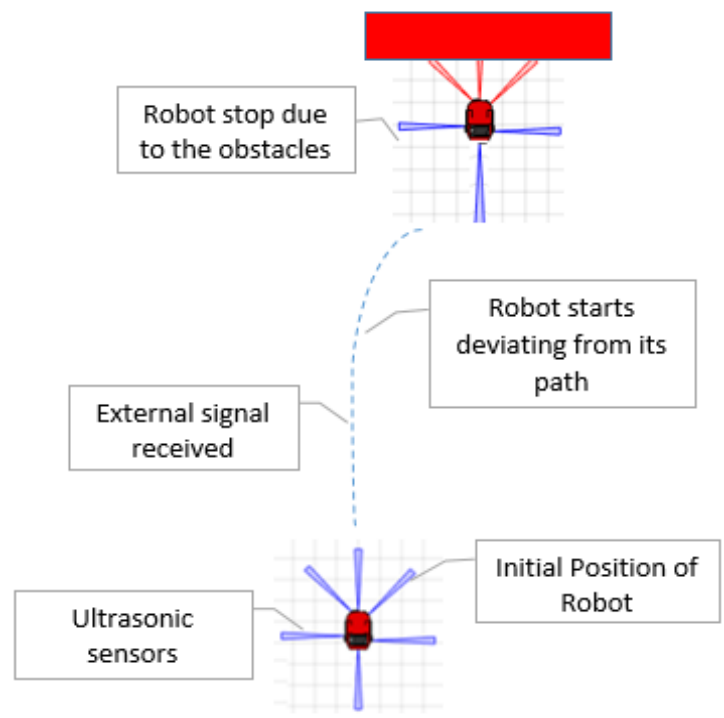

Fig. 6. Robot movement in case of external signal received

An important feature of the robot is to differentiate between the obstacle and target if the user wants to move towards target the robot will move further after reaching safe range till danger range is reached which is very small distance as compared to the safe range.

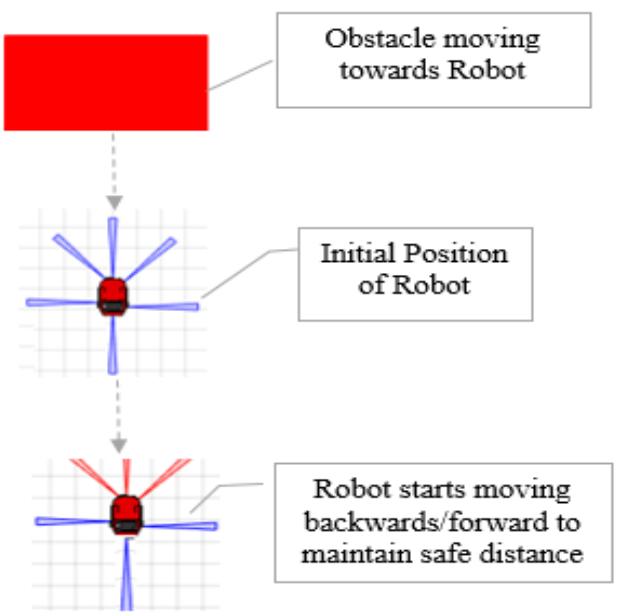

Fig. 7. When obstacle is moving towards robot

After danger range robot will not move any further irrespective of forward signal received. It is also demonstrated in figure 6. When left signal is received from BCI the robot turns left at some radius and continue to move in circle unless next signal is received and when an object comes in safe range the robot stops. As soon as the safe range is reached robot stops irrespective of the signal received from the user. Similar is the case when the right command or signal is received.

\section{B. Moving Obstacle}

In the case when no external signal from the user is received, robot does not move at all, if un-disturbed it behaves 
like an obstacle avoiding robot. If the obstacle is coming towards BCI robot front sensor detects the obstacle and robot starts moving backwards and maintains a safe range from the obstacle similarly if obstacle is following the robot it will move forward and maintains a proper distance from the obstacle which is shown in figure 7.

\section{EXPERIMENTS AND RESULTS}

In order to test the effectiveness of proposed system two experiments were designed. For this, two different paths were designed which differ on the basis of their path complexity. Each experiment had 3 trials. User is directed to go to the specified goal in the described path in the specified time period. Before the actual experiment randomized list of task was designed which included 20 trials of eye movements in order to test the correctness of BCI. The performance is evaluated on the basis of time delay between the eye movements and the execution of command. Workload as well as stress level on the user is also studied by the user feedback at the end of each experiment.

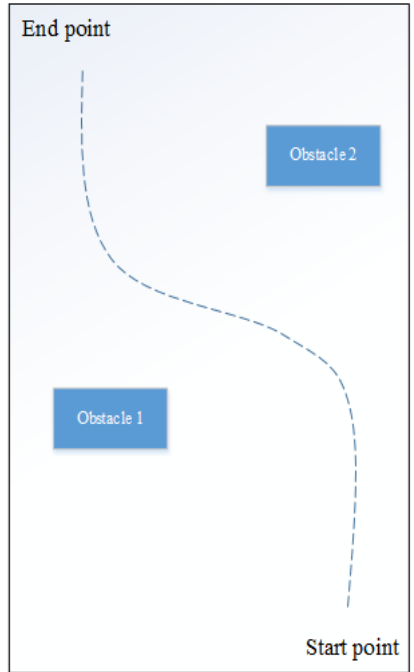

(a)

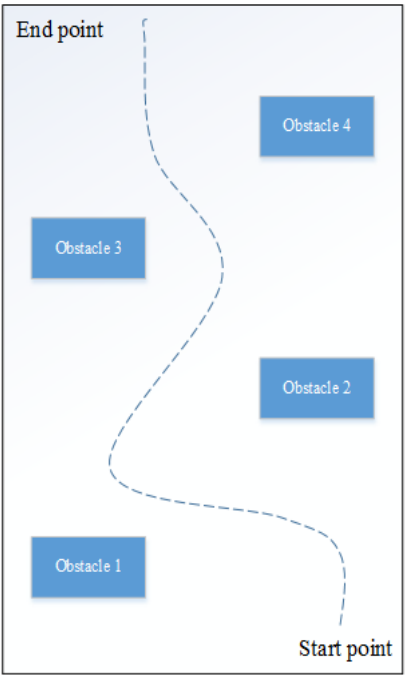

(b)
Fig. 8. (a) Path designed for experiment 1 (b) Path designed for experiment 2

The extent of control of user over robot is another important parameter for the evaluation of proposed system. Speed of the robot has no effect on the BCI but it affects the user in terms of mental workload. At high speed it was difficult for the user to control the robot effectively and it was very tiring and stressful for the user. So the speed of robot was kept low which decreased the workload as well as enabled user to completely control the robot.

In the first experiment only few obstacles were added. The user has to reach the goal by avoiding obstacles. The results showed that the user was fully able to control the robot without any mental workload. While in second experiment a complex path was designed which includes sharp turns and user has to issue more commands in order to reach the goal. Both of the path scenarios are also shown in the figure 8 and the performance evaluation which is on the basis of path 1 versus path 2 is also shown in figure 9 .

\section{Performance evaluation}

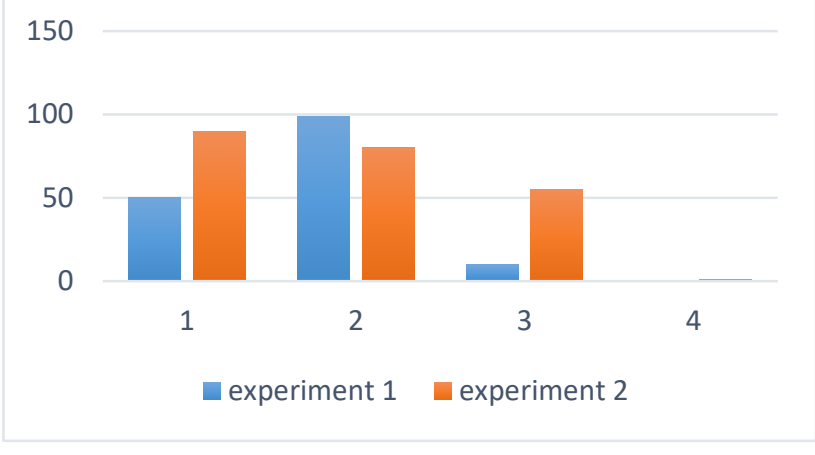

Fig. 9. Performance evaluation

TABLE. I. COMPARISON OF BOTH EXPERIIMENTS

\begin{tabular}{|l|l|l|l|l|}
\hline & $\begin{array}{l}\text { Time to } \\
\text { reach } \\
\text { goal (1) }\end{array}$ & $\begin{array}{l}\text { Control } \\
\text { of robot } \\
\text { (2) }\end{array}$ & $\begin{array}{l}\text { No. of } \\
\text { commands } \\
\text { issued by } \\
\text { user(3) }\end{array}$ & $\begin{array}{l}\text { No. of } \\
\text { collisions } \\
(4)\end{array}$ \\
\hline Experiment 1 & $50 \%$ & $99 \%$ & $30 \%$ & 0 \\
\hline Experiment 2 & $80 \%$ & $80 \%$ & $60 \%$ & 0 \\
\hline
\end{tabular}

The comparative analysis of both experiments is shown in table 1 . The results showed that user was able to reach the specified destination with $80 \%$ accuracy and the time to reach the goal was very high although there were no collisions with the obstacles. The feedback of the user showed that this was a bit tiring and requires more attention of the user. This experiment also showed that this was very safe there were no collision due to the obstacles which suggest that this system can be implemented on wheelchair without any safety issues.

\section{CONCLUSION}

As prototype of brain controlled wheelchair, brain controlled robot was developed. The experiments were conducted in order to test whether the robot moves in accordance with the provided command and the system accuracy was tested by letting a person to control the robot in different path scenarios of variable complexity. The results demonstrated that the robot was successfully controlled by the person using EEG signals from eye movement without a single collision. The shared control is very crucial in this regard and helps the user to avoid any obstacle coming its way. The mental work load from the user is reduced by making the robot autonomous on the basis of last received command which was confirmed by the feedback from the user. However the workload also depends upon the navigation path complexity. The proposed strategy can also be used on industrial robots which are controlled by physically impaired people.

\section{FUTURE WORK}

Authors are working on controlling the robot with different methods which include gestures, muscle activity and other brain signals. Further improvements in this work can also be 
done by implementing a manual control for a third person along with the brain and shared control.

\section{REFERENCES}

[1] J. del R. Millán et al., "Combining brain--computer interfaces and assistive technologies: state-of-the-art and challenges," Front. Neurosci., vol. 4, p. 161, 2010.

[2] B. J. He, "Electrocorticogram (ECoG)," in Encyclopedia of Computational Neuroscience, D. Jaeger and R. Jung, Eds. New York, NY: Springer New York, 2015, pp. 1070-1074.

[3] J. A. Wilson, E. A. Felton, P. C. Garell, G. Schalk, and J. C. Williams, "ECoG factors underlying multimodal control of a brain-computer interface," IEEE Trans. neural Syst. Rehabil. Eng., vol. 14, no. 2, pp. 246-250, 2006.

[4] A. Nijholt et al., "Brain-computer interfacing for intelligent systems," IEEE Intell. Syst., vol. 23, no. 3, 2008.

[5] J. del R. Milan and J. M. Carmena, "Invasive or noninvasive: Understanding brain-machine interface technology [conversations in bme]," IEEE Eng. Med. Biol. Mag., vol. 29, no. 1, pp. 16-22, 2010.

[6] J. R. Wolpaw, D. J. McFarland, G. W. Neat, and C. A. Forneris, "An EEG-based brain-computer interface for cursor control," Electroencephalogr. Clin. Neurophysiol., vol. 78, no. 3, pp. 252-259, 1991.

[7] L. Bi, X.-A. Fan, and Y. Liu, "EEG-based brain-controlled mobile robots: a survey," IEEE Trans. human-machine Syst., vol. 43, no. 2, pp. 161-176, 2013.

[8] M. S. Cardwell, "Locked-in syndrome.," Tex. Med., vol. 109, no. 2, pp. e1--e1, 2013.

[9] R. Golby, B. Poirier, M. Fabros, J. J. Cragg, M. Yousefi, and N. Cashman, "Five-Year Incidence of Amyotrophic Lateral Sclerosis in British Columbia (2010-2015)," Can. J. Neurol. Sci. Can. des Sci. Neurol., pp. 1-5, 2016.

[10] J. A. Müller, D. Wendt, B. Kollmeier, and T. Brand, "Comparing eye tracking with electrooculography for measuring individual sentence comprehension duration," PLoS One, vol. 11, no. 10, p. e0164627, 2016.

[11] R. Barea, L. Boquete, M. Mazo, and E. López, "Guidance of a wheelchair using electrooculography," in Proceeding of the 3rd IMACS International Multiconference on Circuits, Systems, Communications and Computers (CSCC'99), 1999.

[12] S. M. Grigorescu, T. Lüth, C. Fragkopoulos, M. Cyriacks, and A. Gräser, "A BCI-controlled robotic assistant for quadriplegic people in domestic and professional life," Robotica, vol. 30, no. 3, pp. 419-431, 2012.

[13] A. Chella et al., "A BCI teleoperated museum robotic guide," in Complex, Intelligent and Software Intensive Systems, 2009. CISIS'09. International Conference on, 2009, pp. 783-788.

[14] L. Tonin, R. Leeb, M. Tavella, S. Perdikis, and J. del R. Millán, "The role of shared-control in BCI-based telepresence," in Systems Man and Cybernetics (SMC), 2010 IEEE International Conference on, 2010, pp. 1462-1466.

[15] P. Martinez, H. Bakardjian, and A. Cichocki, "Fully online multicommand brain-computer interface with visual neurofeedback using SSVEP paradigm," Comput. Intell. Neurosci., vol. 2007, p. 13, 2007.

[16] E. C. Lalor et al., "Steady-state VEP-based brain-computer interface control in an immersive 3D gaming environment," EURASIP J. Appl. Signal Processing, vol. 2005, pp. 3156-3164, 2005.

[17] L. Mingyu, W. Jue, Y. Nan, and Y. Qin, "Development of EEG biofeedback system based on virtual reality environment," in Engineering in Medicine and Biology Society, 2005. IEEE-EMBS 2005. 27th Annual International Conference of the, 2006, pp. 5362-5364.

[18] F. Galán et al., "A brain-actuated wheelchair: asynchronous and noninvasive brain--computer interfaces for continuous control of robots," Clin. Neurophysiol., vol. 119, no. 9, pp. 2159-2169, 2008.

[19] J. del R. Millán, F. Renkens, J. Mourino, and W. Gerstner, "Noninvasive brain-actuated control of a mobile robot," in Proceedings of the 18th international joint conference on Artificial intelligence, 2003, no. EPFL-CONF-82919.

[20] R. Leeb, D. Friedman, G. R. Müller-Putz, R. Scherer, M. Slater, and G. Pfurtscheller, "Self-paced (asynchronous) BCI control of a wheelchair in virtual environments: a case study with a tetraplegic," Comput. Intell. Neurosci., vol. 2007, 2007.

[21] I. A. Mirza et al., "Mind-controlled wheelchair using an EEG headset and arduino microcontroller," in Technologies for Sustainable Development (ICTSD), 2015 International Conference on, 2015, pp. 15 .

[22] R. Zhang, Y. Li, Y. Yan, H. Zhang, and S. Wu, "An intelligent wheelchair based on automated navigation and BCI techniques," in Engineering in Medicine and Biology Society (EMBC), 2014 36th Annual International Conference of the IEEE, 2014, pp. 1302-1305.

[23] R. Leeb, L. Tonin, M. Rohm, L. Desideri, T. Carlson, and J. del R. Millán, "Towards independence: a BCI telepresence robot for people with severe motor disabilities," Proc. IEEE, vol. 103, no. 5, pp. 969 982, 2015.

[24] A. Frisoli et al., "A new gaze-BCI-driven control of an upper limb exoskeleton for rehabilitation in real-world tasks," IEEE Trans. Syst. Man, Cybern. Part C (Applications Rev., vol. 42, no. 6, pp. 1169-1179, 2012.

[25] J. Webb, Z. G. Xiao, K. P. Aschenbrenner, G. Herrnstadt, and C. Menon, "Towards a portable assistive arm exoskeleton for stroke patient rehabilitation controlled through a brain computer interface," in Biomedical Robotics and Biomechatronics (BioRob), 2012 4th IEEE RAS \& EMBS International Conference on, 2012, pp. 1299-1304.

[26] S. W. Wijesoma, D. F. H. Wolfe, and R. J. Richards, "Eye-to-hand coordination for vision-guided robot control applications," Int. J. Rob. Res., vol. 12, no. 1, pp. 65-78, 1993.

[27] M. Lin, B. Li, and Q.-H. Liu, "Identification of eye movements from non-frontal face images for eye-controlled systems," Int. J. Autom. Comput., vol. 11, no. 1, pp. 543-554, 2014.

[28] J. P. Hansen, A. W. Andersen, and P. Roed, "Eye-gaze control of multimedia systems," Adv. Hum. Factors/Ergonomics, vol. 20, pp. $37-$ 42, 1995.

[29] C. H. Morimoto and M. R. M. Mimica, "Eye gaze tracking techniques for interactive applications," Comput. Vis. image Underst., vol. 98, no. 1, pp. 4-24, 2005.

[30] A. Vallabhaneni, T. Wang, and B. He, "Brain-computer interface," in Neural engineering, Springer, 2005, pp. 85-121.

[31] R. Zhang et al., "Control of a Wheelchair in an Indoor Environment Based on a Brain--Computer Interface and Automated Navigation," IEEE Trans. neural Syst. Rehabil. Eng., vol. 24, no. 2, pp. 128-139, 2016.

[32] I. Iturrate, J. M. Antelis, A. Kubler, and J. Minguez, "A noninvasive brain-actuated wheelchair based on a P300 neurophysiological protocol and automated navigation," IEEE Trans. Robot., vol. 25, no. 3, pp. 614627, 2009.

[33] B. Rebsamen et al., "A brain controlled wheelchair to navigate in familiar environments," IEEE Trans. Neural Syst. Rehabil. Eng., vol. 18, no. 4, pp. 590-598, 2010. 